\title{
Novel Technologies and Theoretical Models in Radiation Therapy of Cancer Patients Using 6.3 MeV Fast Neutrons Produced by U-120 Cyclotron
}

\author{
L. I. Musabaeva ${ }^{1, a)}$, Zh. A. Startseva ${ }^{1,2, b)}$, O. V. Gribova ${ }^{1,2, c)}$, \\ V. V. Velikaya ${ }^{1,2, \mathrm{~d})}$, and V. A. Lisin ${ }^{1, \mathrm{e})}$ \\ ${ }^{1}$ Tomsk Cancer Research Institute, Kooperativny Street 5, Tomsk, 634050 Russia \\ ${ }^{2}$ National Research Tomsk Polytechnic University, Lenin Avenue 30, Tomsk, 634050 Russia

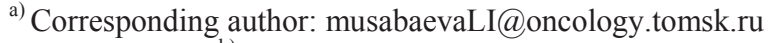 \\ b) zhanna.alex@rambler.ru \\ c) gribova79@mail.ru \\ d) viktoria.v.v@inbox.ru \\ e)Lisin@oncology.tomsk.ru
}

\begin{abstract}
The analysis of clinical use of neutron therapy with $6 \mathrm{MeV}$ fast neutrons compared to conventional radiation therapy was carried out. The experience of using neutron and mixed neutron and photon therapy in patients with different radio-resistant malignant tumors shows the necessity of further studies and development of the novel approaches to densely-ionizing radiation. The results of dosimetry and radiobiological studies have been the basis for planning clinical programs for neutron therapy. Clinical trials over the past 30 years have shown that neutron therapy successfully destroys radio-resistant cancers, including salivary gland tumors, adenoidcystic carcinoma, inoperable sarcomas, locally advanced head and neck tumors, and locally advanced prostate cancer. Radiation therapy with $6.3 \mathrm{MeV}$ fast neutrons used alone and in combination with photon therapy resulted in improved long-term treatment outcomes in patients with radioresistant malignant tumors.
\end{abstract}

\section{INTRODUCTION}

Neutron therapy is used alone or in combination with photon therapy. The dependence of the frequency of regression of primary, recurrent and metastatic head and neck tumors and morphological characteristics of the tumor as well as contribution of fast neutrons to the total dose has been established. In neutron and neutron-photon therapy, the character and the rate of reoxygenation of superficial tumors was found to depend on the size of the primary tumor. In this context, the radiation therapy regimens based on the use of mixed gamma-neutron irradiation allow the group of cancer patients to be significantly expanded. Studies on the activity of the saliva enzymes including alpha-amelase, kallikrein and kallikreinogen were carried out for the first time in patients with oral cavity cancer, who underwent neutron and neutron-photon therapy. Suppression of enzymatic activity of saliva was more clearly pronounced in patients subjected to neutron therapy than in patients subjected to the conventional gamma-therapy. Indices of saliva enzymatic activity in the process of combined radiotherapy were used as a criterion of tolerance of salivary glands in the patients with head and neck cancer. Copper steam laser irradiation, magnetic therapy and ozone therapy have been shown to be useful for the treatment and prevention of radiation-induced skin damage in the long-term follow-up of patients with breast cancer local recurrence. Thus, due to the novel radiotherapy technologies, $6.3 \mathrm{MeV}$ fast neutrons produced by U-120 cyclotron have been used for the treatment of cancer patients at the Tomsk Cancer Research Institute for more than 30 years. 


\section{DOSIMETRIC AND RADIOBIOLOGICAL CHARACTERISTICS OF NEUTRON AND NEUTRON-PHOTON THERAPY}

All regimens of neutron and mixed neutron-photon therapy are based on radiobiological and mathematical models. The time, dose and fractionation (TDF) model was applied first, then the linear quadratic model (LQM) was used to measure spatial neutron dose distribution and RBE of fast neutrons. The radiobiological properties of a cyclotron-produced fast neutron beam relative to gamma rays allow us to consider neutron therapy as one of the ways to improve radiation therapy results.

A computer program for measuring spatial dose distribution in neutron and mixed gamma-neutron therapies has been created, allowing the total distribution of the absorbed and isoeffective doses for the mixed gamma-neutron therapy to be calculated [1]. The main advantage of new regimens is an 8-fold increase in the frequency of breast cancer local recurrence and increase in the 5-year disease-free survival rate of patients with various types of cancer. The use of the TDF and LQ models contributes to the prediction of radiation-induced damage to normal tissues.

\section{NEUTRON THERAPY REGIMENS}

The following two neutron therapy regimens were used: (1) 2 fractions per week, 1.2-1.4 Gy single dose (3.23.1 RBE), 5.3-4.5 Gy photon-equivalent dose, 40-60 Gy total dose, for 4-6 weeks; (2) 3 fractions with a 4872 hour interval, 1.8-2.4 Gy single dose (2.6-2.8 RBE), 6.7-6.8 Gy photon-equivalent dose, 38-40 Gy total dose, for 8-12 days. It should be noted that the applied regimens of fast neutron dose fractionation, particularly in the regime 2, when only three 1.8-2.4 Gy fast neutrons fractions were used, represent enlarged single dose version.

These regimens were used for preoperative and postoperative neutron therapy alone and in combination with external beam radiotherapy for both curative and palliative cancer treatment. Results of clinical trials demonstrated that neutron therapy was well tolerated, and there was no evidence of severe radiation induced reactions.

\section{RESULTS AND DISCUSSION}

The method of neutron-photon therapy for locally advanced breast cancer was developed (RF patent No. 2186591) [2]. To assess the efficacy of preoperative neutron therapy, the treatment regimen 2 (3 fractions, 2.62.8 RBE, 6.7-6.8 Gy photon-equivalent dose) was used. Normal and tumor tissue pathological response was assessed after radical mastectomy. The histological study showed that fibrous tissue of different maturity predominated both in the centre and on the periphery of the tumor [3]. Tumor cells persisted in groups and individually, but almost in $100 \%$ of the cases they were degenerately changed. The number of normal mitoses in tumors was minimal $(20.88 \pm 2.0 \%)$, while the number of pathologic mitoses was maximum $(79.12 \pm 2.8 \%)$. The greatest number of "lethal mitoses" was observed in tumors after neutron therapy and the minimum number after the conventional photon therapy, thereby indicating that $6.3 \mathrm{MeV}$ fast neutron therapy resulted in a significant damage to tumor cells compared to the standard photon therapy. The replacement of the tumor with fibrous tissue of various degrees of maturity was typical of preoperative neutron therapy for breast cancer. The neutron therapy regimen consisting of 3 fractions resulted in a long-term disease-free follow-up period in patients with locally advanced cancer. Late radiation-induced damage to normal tissues was corresponded to grade 1 toxicity (according to the Radiation Therapy Oncology Group/The European Organization for Research, and Treatment of Cancer (RTOG/ EORTC).

Forty-one patients with head and neck cancer and 44 patients with musculoskeletal cancer underwent preoperative neutron and mixed neutron-photon therapies followed by surgery. No complications in the postoperative period were observed in cases when the total dose of neutron radiation therapy was $\leq 40 \mathrm{~Gy}$. The increase in the absorbed neutron dose or the prolonged follow-up period led to the growth in the frequency of postoperative complications. Histological examination of surgical specimen showed that the microscopic features of radiationinduced damage to tumor tissue were similar both after neutron and mixed neutron-photon radiation therapies. Radiation-induced damages were manifested as degenerative changes, necrobiosis and necrosis of parenchymal cells. Neutron therapy contributed to the reduction in the tumor mitotic index, number of normal mitoses and number of cells emerging from mitoses. There was a growing level of pathological mitoses, including scattering, chromosome fragmentation, asymmetry and K-mitoses. The DNA accumulation index was 2.48 after preoperative neutron therapy and 4.6 after photon therapy. Response of osteogenic sarcoma to neutron and mixed neutron-photon therapy differed by more marked changes in comparison with that to the conventional photon therapy. 
Preoperative neutron therapy was given to 46 patients with cancer of the maxillary sinus and nasal cavity. The control group comprised 45 patients who received the conventional photon therapy. The overall 5 -year survival rates were: $62 \pm 9.6 \%$ in the neutron therapy group and $42 \pm 9.5 \%$ in the control group. The 5-year recurrence-free survival rates were $68 \pm 8.2 \%$ and $40 \pm 9.2 \%$, respectively. At a follow-up of 10 years, 11 of the 38 patients $(29 \%)$ are still alive in the neutron therapy group and none of the patients $(0 \%)$ is alive in the control group.

Results of postoperative neutron therapy for patients with parotid gland cancer $(n=52)$ were as follows: the 5-year relapse-free survival rate was $72.4 \pm 9.8 \%$ and the 10 -year survival rate was $32.2 \pm 16.8 \%$. In the control group the 5-year survival rate was $25.6 \pm 19.1 \%(p<0.05)$. In patients with salivary gland cancer $(n=22)$, the 3 and 5-year survival rates were $60.6 \pm 13.6 \%$ and $48.5 \pm 15.3 \%$, respectively after neutron therapy compared to $30 \pm 16.9 \%$ and $0 \%$ after photon therapy $(p<0.05)$. In patients with thyroid cancer $(n=33)$, the overall 5 -year- and relapse-free survival rates were $70.4 \pm 7 \%$ and $72.4 \pm 9.8 \%$, respectively after postoperative neutron therapy compared to $40 \%$ after combined neutron and photon therapy.

The method of radiation therapy with fast neutrons and neutron + photon therapy for 95 patients with breast cancer local recurrence was developed [4]. The overall 6-year survival rate was $92.2 \pm 5.7 \%$ versus $51.5 \pm 9.1 \%$ in the control group. Late radiation-induced skin damages were observed in $23 \%$ of cases with local recurrences. The incidence and character of acute and late radiation-induced skin reactions in breast cancer patients after treatment with preoperative neutron and photon therapy are shown in Fig. 1 and Table 1.

In patients with salivary gland cancer, who received combination treatment with postoperative neutron therapy, the 3-, 5-and 10-year survival rates were $70.4 \pm 7.8 \%, 64.5 \pm 9.1 \%$ and $32.2 \pm 16.8 \%$, respectively. In the control group patients, the 3-year overall survival rate was $51.1 \pm 12.8 \%$ and the 5-year survival rate was $25.6 \pm 19.1 \%$.

The 3- and 5-year overall survival rates in patients with inoperable salivary gland cancer, who received fast neutron therapy alone, were $60.6 \pm 13.6 \%$ and $48.5 \pm 15.3 \%$, respectively. In the control group, the corresponding values were $30 \pm 16.9 \%$ and $0 \%$, respectively [5]. The 3 - and 5-year recurrence-free survival rates were $80.4 \pm 6.8 \%$ and $72.4 \pm 9.8 \%$ for salivary gland cancer patients treated with surgery and postoperative neutron therapy, respectively. In the control group, the 3- and 5-year recurrence-free survival rate was $42.4 \pm 18.6 \%$.

\section{Incidence of acute and late radiation-induced skin reactions in breast cancer patients after treatment with preoperative neutron and photon therapy (scale RTOG/EORTS, 1995)}

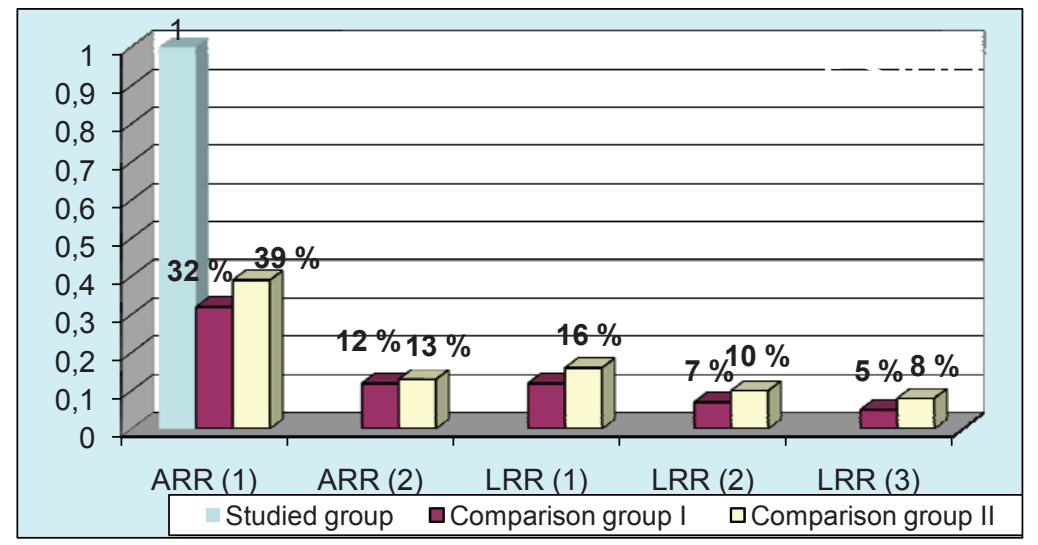

Post-radiation reaction [1] - Slight atrophy, change in skin pigmentation, mild epilation, slight induration (fibrosis) and decrease in the amount of subcutaneous fat

Post-radiation reaction[2] -Island atrophy, moderate telangiectasis, moderate symptomatic-free fibrosis in the radiation area

Post-radiation reaction [3] - Severe atrophy, massive telangiectasis, noticeable induration and decrease in the amount of subcutaneous fat

FIGURE 1. Incidence and character of acute and late radiation-induced skin reactions in breast cancer patients after treatment with preoperative neutron and photon therapy (scale RTOG/EORTS, 1995) 
TABLE 1. Short-term treatment results of radiation therapy for patients with breast cancer local recurrences with respect to the type of ionizing radiation

\begin{tabular}{lccc}
\hline & \multicolumn{3}{c}{ Number of patients, \%) } \\
\cline { 2 - 4 } Treatment response & $\begin{array}{c}\text { Neutron therapy, } \\
\text { total radiation dose } \\
\text { is } \begin{array}{c}\text { 40-60 isoGy } \\
(n=7)\end{array}\end{array}$ & $\begin{array}{c}\text { Neutron-photon } \\
\text { therapy, } \\
\text { total radiation dose } \\
\text { is } 60-65 \text { isoGy } \\
(n=40)\end{array}$ & $\begin{array}{c}\text { Electron therapy, } \\
\text { total radiation dose is } 60-65 \\
\text { isoGy }(n=41)\end{array}$ \\
\hline Complete response & $7(100 \%)$ & $37(93 \%)$ & $25(61 \%)$ \\
\hline Partial response & - & $3(7 \%)$ & $5(12 \%)$ \\
\hline Stable disease & - & - & $11(27 \%)$ \\
\hline Disease progression & - & - & - \\
\hline
\end{tabular}

Immediate response rate of inoperable salivary gland cancer was higher to fast neutrons than to conventional photon therapy. In neutron therapy group, complete tumor regression was observed in 23 cases $(58 \%)$, partial regression in 15 cases $(37 \%)$. No response to therapy with evidence of disease progression was noted in two cases $(5 \%)$. In the control group, partial tumor regression was observed in 5 cases $(62.5 \%)$. No response to radiotherapy was recorded in 2 cases $(25 \%)$, complete regression occurred in one case $(12.5 \%)$.

The analysis of long-term treatment outcomes of patients with thyroid cancer showed the overall five-year survival rate of $32.8 \pm 15.1 \%$ in patients with anaplastic thyroid carcinoma and $63.4 \pm 7.8 \%$ in patients with medullary carcinoma [6]. All patients with squamous cell carcinoma of the thyroid gland died of distant metastases without evidence of local recurrence within 1-year follow-up.

Radiation-induced skin damages (mainly grade I erythema according to RTOG scale) were observed in $81 \%$ of patients with salivary gland cancer who received combination treatment including surgery and postoperative neutron or neutron-photon therapy [7]. In patients with salivary gland cancer, who received neutron or neutron-photon therapy alone, radiation-induced skin damages were noted in $96 \%$ of cases.

In patients with thyroid cancer, who were treated with fast neutrons in the postoperative period, radiationinduced skin reactions were observed in $73 \%$ of cases and were mainly represented by erythema (52\%). In patients with thyroid cancer, who received neutron-photon therapy alone, dry and moist desquamation was observed in $81 \%$ of cases.

\section{CONCLUSION}

The rates of recurrence-free and overall survival for patients with radio-resistant cancer treated with combined modality treatment including fast neutron therapy were significantly higher than those observed in patients treated with conventional photon therapy. The assessment of $6.3 \mathrm{MeV}$ fast neutron therapy regimens in terms of tumor response and overall disease-free survival indicate the advantage of fast neutron therapy over the conventional photon therapy in patients with radio-resistant malignant tumors. Clinical trials of neutron-photon therapy used alone and in combination with surgery for cancer patients have shown feasibility of increasing the effectiveness of treatment due to reduction in the incidence of local recurrences. In addition, satisfactory treatment tolerance and absence of severe specific complications dictate the necessity of prospective studies to improve treatment outcomes. The accumulated experience in the use of neutron and neutron-photon therapies for patients with radio-resistant cancer has shown that further development of this modality is necessary to achieve the best outcomes for our patients.

\section{ACKNOWLEDGMENTS}

The study was financially supported by the Fundamental Research Program of the Russian Academy of Sciences, project No. 093. 
The study reported in this article was conducted according to accepted ethical guidelines involving research in humans and/or animals and was approved by an appropriate institution or national research organization.

The study is compliant with the ethical standards as currently outlined in the Declaration of Helsinki.

All individual participants discussed in this study, or for whom any identifying information or image has been presented, have freely given their informed written consent for such information and/or image to be included in the published article.

\section{REFERENCES}

1. V. A. Lisin, Dosimetry computer therapy planning of malignant neoplasms by fast neutron beam of U-120 cyclotron, Medical Radiology 1, 26-28.

2. L. I. Musabaeva, V. A. Lisin, and E. M. Slonimskaya, The method of neutron photon therapy of locally advanced breast cancer, RF Patent, No. 2186591.

3. M. F. Halva, Morphological Criteria of Efficiency, Chapter 4.4 (Publishing House of Scientific and Technical Literature, Tomsk, 2000), p. 188.

4. V. V. Velikaya, Efficiency of the neutron and neutron-photon therapy in complex treatment of local recurrence of breast cancer, PhD thesis, Tomsk, 2007.

5. P. M. Speight and A. W. Barrett, Salivary gland tumours, Oral Dis. 8(5), 229-240 (2002).

6. V. A. Lisin, Theoretical evaluation of the effectiveness of tumors fractionated radiation by fast neutrons, Radiology and Nuclear Medicine 10, 13-18 (1986).

7. V. A. Lisin and L. I. Musabaeva, Quantitative assessment of radiation-induced reactions with regard to their radiobiological parameters, Medical Radiology 28(12), 30-34 (1983). 